\title{
Regulation of activin type I receptor function by phosphorylation of residues outside the GS domain
}

\author{
Susan A. Willis, Lawrence S. Mathews* \\ Department of Biological Chemistry, University of Michigan, 1301 Catherine Rd., Ann Arbor, MI 48109-0606, USA
}

Received 5 November 1997; revised version received 20 November 1997

\begin{abstract}
Activin signals through a heteromeric complex of receptor serine kinases by inducing type II receptor-mediated phosphorylation, and consequent activation, of the type I receptor. Type I receptor phosphorylation occurs at a glycineand serine-rich site in the juxtamembrane domain; phosphorylation at that site correlates with signaling. Investigation of type I activin receptor mutants impaired for GS domain phosphorylation revealed that, in the presence of elevated amounts of type II activin receptor, GS domain phosphorylation is not required for signaling. The type I receptor showed activin-dependent phosphorylation of several tryptic phosphopeptides, suggesting that phosphorylation of receptor $I$ at sites both within and outside the GS domain is required for full signaling.
\end{abstract}

(C) 1997 Federation of European Biochemical Societies.

Key words: Activin; Receptor serine kinase;

Protein phosphorylation

\section{Introduction}

The receptor serine kinase family contains two classes of transmembrane protein serine kinases, the type I receptors and the type II receptors [1]. Members of this family mediate transmembrane signaling for activin, transforming growth factor $\beta$ (TGF $\beta$ ) and related factors, and are thought to act by formation of a heterooligomeric complex containing both type I and type II receptors. A current model for receptor action posits that ligand binding induces the formation of a type I:type II complex, resulting in type II receptor-mediated activation of the type I receptor by phosphorylation at residues within a conserved glycine- and serine-rich domain in the juxtamembrane region [2]. For activin [3,4] and TGF $\beta$ [5] type I receptors (ALK4 and T $\beta$ RI, respectively), mutation to alanine of the hydroxyamino acids in that GS domain yields molecules which can no longer support ligand-dependent transcriptional activation, implying that such phosphorylation is required for normal signal transduction to occur. The mechanism through which modification of residues within the GS domain enhances signaling by the type I receptors, either by stimulation of intrinsic enzymatic activity or by recruitment of intracellular signaling molecules, is not currently known.

In an earlier study of the ALK4 GS domain, we observed that potential phosphorylation of some GS domain residues was functionally more significant than others [3]. Specifically, certain mutants in which four out of five hydroxyamino acids in the GS domain were changed to alanine retained significant signaling capacity. In pursuing the correlation of phosphorylation with function, we have now found that under conditions

*Corresponding author. Fax: (1) (313) 763-4581.

E-mail: lmathews@umich.edu of type II activin receptor (ActR) overexpression, phosphorylation at the GS domain is not absolutely required to generate an activin-dependent transcriptional response.

\section{Materials and methods}

\subsection{Materials}

Purified activin A was generously provided by Dr. Yuzuru Eto, and by the National Hormone and Pituitary Program, the NIDDK, NICHD, and USDA (lot 15365-36). D-Luciferin was obtained from Sigma Chemical Co (St. Louis, MO). [ $\left.{ }^{32} \mathrm{P}\right]$ Orthophosphate $(500 \mathrm{mCi} /$ $\mathrm{ml}$ ) was obtained from ICN Pharmaceuticals (Irvine, CA). Activin receptor (ActR) DNAs and p3TP-Lux were as described [3]

\subsection{Luciferase assay}

The L17 mink lung epithelial cell line [6] was cultured and transfected as described [3]. For luciferase assays, L17 cells were plated at a density of $2.5 \times 10^{4}$ cells per well in 24 -well plates and grown overnight before transfection. Cells were transfected with $0.5 \mu \mathrm{g}$ of the p3TP-Lux reporter plasmid and combinations of ActR DNAs $(0.1 \mu \mathrm{g})$ as indicated. Cells were co-transfected with $0.25 \mu \mathrm{g}$ of a plasmid expressing $\beta$-galactosidase. Following transfection, cells recovered for 30 $\mathrm{h}$ and were then stimulated with $2 \mathrm{nM}$ activin $\mathrm{A}$ in $0.25 \mathrm{ml}$ medium containing $0.2 \%$ fetal bovine serum (v/v). Luciferase activity was measured in $40 \mu \mathrm{l}$ of a $200 \mu \mathrm{l}$ lysate as described [3]. Luciferase activity was normalized to $\beta$-galactosidase activity measured in 20 $\mu 1$ of lysate as described [7]. The data in Figs. 1-3 show results from single representative experiments; each experiment was repeated at least three times.

\subsection{Phosphorylation analysis}

Cells plated in six-well dishes (approximately $2 \times 10^{5}$ cells per well) were transfected with various receptor combinations and allowed to recover in complete medium overnight. The transfected cells were labeled with $\left[{ }^{32} \mathrm{P}\right]$ orthophosphate, and ALK4 proteins were immunoprecipitated with anti-HA antibody [8] exactly as described [3]. Tryptic phosphopeptide mapping and phosphoamino acid analysis were performed exactly as described for ActRII $[9,10]$.

\section{Results and discussion}

In a previous report [3] we noted that one of three tested quadruple mutants of the ALK4 GS domain (ALK4-4A-S187, in which serine 187 was the only one of the five serine or threonine residues in the GS domain not mutated) had the capacity to support activin responsiveness in receptor I-deficient cells (see Fig. 1). To follow up that observation, all five quadruple mutants of ALK4 were prepared and tested for their capacity to confer activin inducibility of the 3TP-Lux reporter gene in L17 cells. In addition to ALK4-4A-S187, ALK4-4A-S189 and ALK4-4A-S191 also mediated activin signaling (Fig. 1). Interestingly, S187, S189 and S191 were the only residues which when mutated in combination of three rendered ALK4 inactive in this assay [3] (see Fig. 2, ATAAS), consistent with the interpretation that phosphorylation of those specific residues is associated with signaling. 


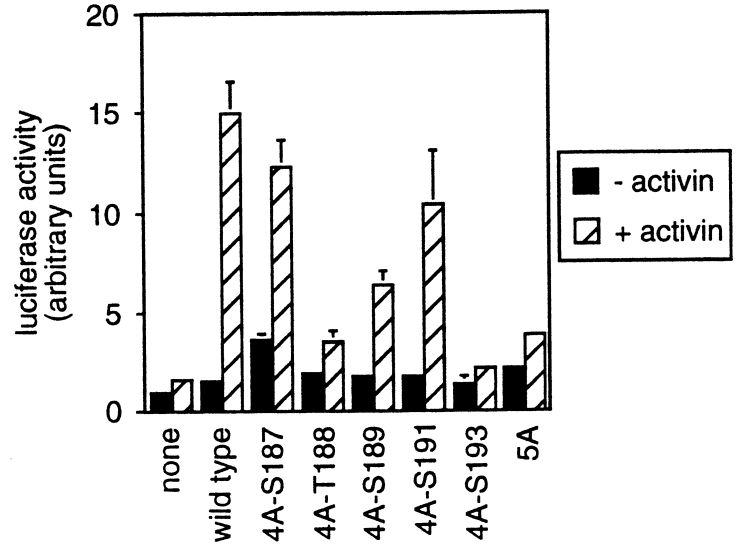

Fig. 1. Signaling activity of ALK4 GS domain mutants. L17 cells were transfected with 3TP-Lux together with different ALK4 constructs as indicated. Cells were treated with $2 \mathrm{nM}$ activin $\mathrm{A}$ and then luciferase and $\beta$-galactosidase activity were measured. The data were normalized with $\beta$-galactosidase activity, and the level of luciferase expression in the absence of exogenous receptors and the absence of activin set to one. Values were determined in triplicate and represent the mean \pm standard deviation. The data are representative of several independent experiments.

We also showed previously that ALK4-ETEES (S187E, S189E, S191E) had a signaling capacity similar to wild-type. To determine whether that signaling was potentially due to residual phosphorylation of the other residues in the GS domain, we prepared and tested ALK4-EAEEA, which also signaled normally (Fig. 2). This result supports our earlier conclusion that acidic substitutions of specific hydroxyamino acids in the ALK4 GS domain can mimic functional phosphorylation; however, because basal signaling remained low these data imply that additional activin-dependent regulation of ALK4 is required for full activity (see below).

Because L17 cells express very low amounts of type II ActRs as judged by ligand binding experiments (not shown), we determined the effect of overexpression of ActRIIB together with different GS domain mutants. Fig. 3 shows representative results for a partially active quadruple mutant (4AS189), an inactive quadruple mutant (4A-S193), and the non-phosphorylatable mutant ALK4-5A. Surprisingly, expression of elevated amounts of ActRIIB increased the activin responsiveness conferred on the cells by all the ALK4 mutants tested, including ALK4-5A. For 4A-S189, the change in activin induction was increased from $3-5$ fold in the absence of ActRIIB to 10-20 fold in the presence of ActRIIB; for 4AS193 and 5A, the corresponding induction went from approximately 1.5 fold to 3-8 fold. As previously observed, overexpression of ActRIIB alone neither affected basal expression of the reporter gene nor rendered the cells responsive to activin. Both ActRII and ActRIIB had this ALK4-potentiating activity, while T $\beta$ RII did not (not shown). The kinase activity of ActRIIB was essential to bring about this rescue of ALK4 signaling, as an enzymatically inactive mutant (ActRIIBK217R) was unable to induce an activin response (Fig. 3).

It is possible that GS domain phosphorylation markedly increases ALK4 activity, but that ALK4 mutants which are not phosphorylated at the GS domain do have very low basal signaling activity when complexed with type II receptors. Because receptor II is present only at very low amounts in L17 cells, that low amount of signaling is insufficient to generate a

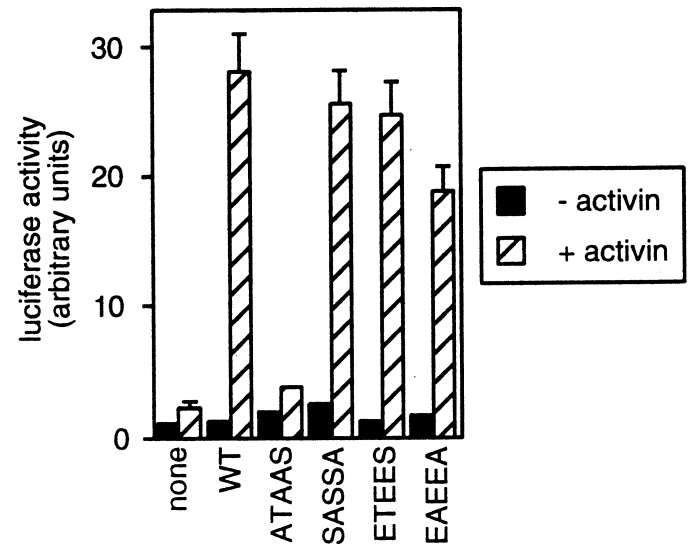

Fig. 2. Replacement of ALK4 GS domain phosphorylation sites with acidic amino acids. L17 cells were transfected with 3TP-Lux together with the indicated ALK4 mutants. WT, wild-type GS domain sequence (STSGSGS, S187, T188, S189, S191, S193). Cells were treated with activin and assayed as in Fig. 1.

biological response from ALK4-5A. Quantitatively increasing the number of receptor complexes formed - by overexpression of ActRIIB - could thus lead to enough of an increase in the number of weakly signaling ALK4-5A:ActRIIB complexes to allow for a modest response to activin treatment. Given that type II receptor kinase activity was required for this response, the enhanced ALK4 activity is likely due to increased phosphorylation at a site(s) distinct from the GS domain.

To examine ALK4 phosphorylation, ALK4 and ALK4-5A were transiently expressed in L17 cells together with ActRIIB, metabolically labeled with $\left[{ }^{32} \mathrm{P}\right]$ orthophosphate, and analyzed

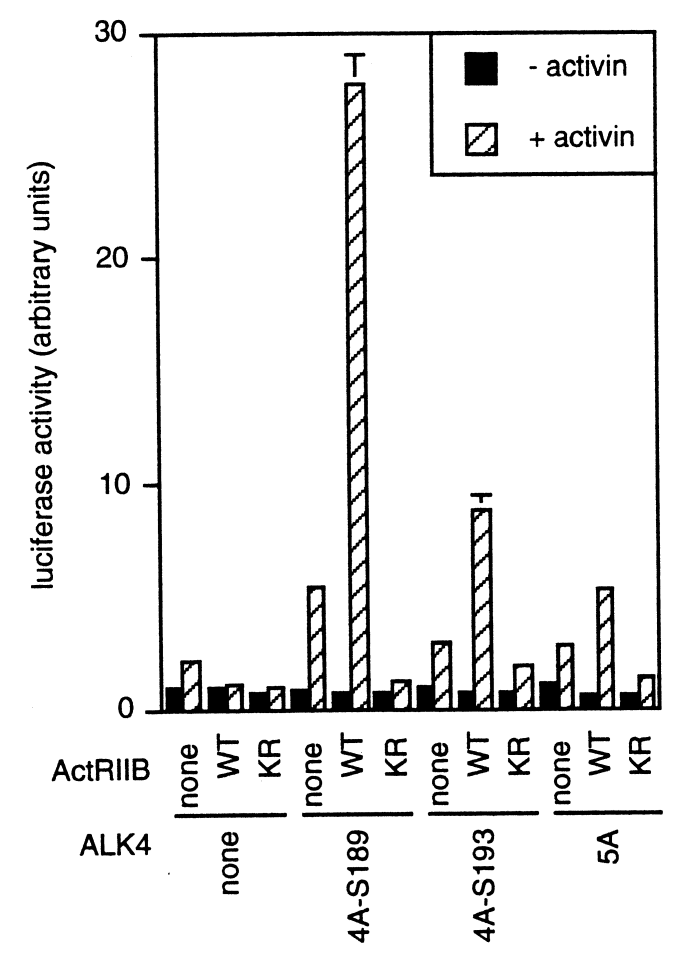

Fig. 3. Effect of type II activin receptor on type I receptor signaling. L17 cells were transfected with 3TP-Lux together with combinations of different type I and type II receptor constructs as indicated. Cells were treated with activin and assayed as in Fig. 1. WT, wild-type ActRIIB; KR, ActRIIB-K217R, an enzymatically inactive point mutant. 

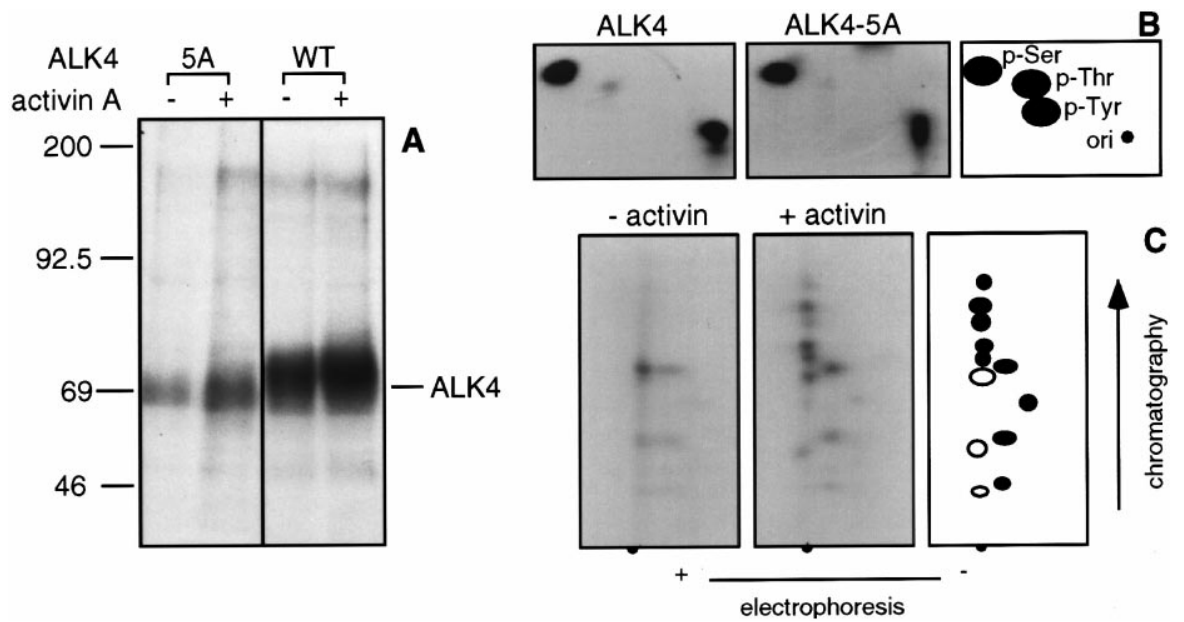

Fig. 4. Phosphorylation of ALK4. A: L17 cells were transfected with HA-tagged ALK4 or ALK4-5A together with ActRIIB, metabolically labeled with $\left[{ }^{32} \mathrm{P}\right]$ orthophosphate, incubated for $10 \mathrm{~min}$ with or without $2 \mathrm{nM}$ activin $\mathrm{A}$ as indicated, and immunoprecipitated with the anti-HA antibody, 12CA5. Proteins were resolved on 10\% SDS-PAGE, transferred to a PVDF membrane, and visualized by autoradiography. Molecular mass markers are indicated in $\mathrm{kDa}$. B: Phosphorylated proteins from A were excised from the filter, hydrolyzed with $6 \mathrm{~N} \mathrm{HCl}$ and the resulting phosphoamino acids were resolved in two dimensions by thin-layer electrophoresis (10). (C) Tryptic phosphopeptide maps of ALK4. Phosphorylated ALK4 bands, with and without activin treatment as indicated, were excised from the PVDF membrane, digested with trypsin and eluted from the filter, and the resulting phosphoamino acids were resolved in two dimensions by thin-layer electrophoresis [10]. C: Tryptic phosphopeptide maps of ALK4. Phosphorylated ALK4 bands, with and without activin treatment as indicated, were excised from the PVDF membrane, digested with trypsin and eluted from the filter, and the phosphopeptides were resolved by thin-layer electrophoresis in the first dimension and ascending chromatography in the second dimension. The right panel represents a schematic of the patterns; open and filled spots denote peptides observed in the absence and presence of activin, respectively.

by immunoprecipitation. Although both proteins were expressed in comparable amounts (not shown), ALK4 was phosphorylated to a substantially greater extent than was ALK4-5A in both the basal and ligand-activated states (Fig. $4 \mathrm{~A})$. The reduced phosphorylation observed in ALK4-5A is consistent with the wild-type receptor undergoing modification at the GS domain. Phosphoamino acid analysis of those samples revealed that ALK4 was phosphorylated primarily on serine, with a minor component on threonine; phosphotyrosine was not detected (Fig. 4B). Phosphothreonine was not detected in ALK4-5A, suggesting that T188 in the GS domain is phosphorylated in the wild-type protein; curiously, T188 is not one of the residues implicated by the mutagenic analysis as required for signaling. As previously observed [3], activin treatment resulted in a modest increase in ALK4 phosphorylation; an increase in phosphorylation of ALK4-5A was also seen (Fig. 4A).

To visualize those phosphorylation sites, ${ }^{32} \mathrm{P}$-labeled proteins were digested with trypsin and resolved in two dimensions on thin-layer chromatography plates. For wild-type ALK4, a number of novel tryptic phosphopeptides were detected in the activin-treated sample that were not present in the basal state (Fig. 4C). Although the identities of those various peptides are not currently known, it is likely that some of them represent phosphorylation at the GS domain and some represent phosphorylation of other sites. The GS domain is contained on a single tryptic phosphopeptide; however, different phosphoisomers would have different mobilities under these conditions. Given the reduced amount of phosphorylation of ALK4-5A, clear tryptic phosphopeptide maps have not been generated for that receptor; however, the increase in overall phosphorylation in response to activin treatment is consistent with the interpretation that ligand-regulated phosphorylation occurs at non-GS domain sites.

Taken together, the following observations support the no- tion that phosphorylation of activin type I receptor must occur at both the GS domain and additional residues to achieve full activation. (1) ALK4-5A, which cannot be phosphorylated at the GS domain, can support activin signaling in the presence of a sufficient amount of type II receptor. (2) Acidic substitution of key residues in the GS domain can apparently mimic phosphorylation at those sites, but is insufficient to generate a constitutively active receptor. (3) Analysis of ALK4 and ALK4-5A phosphorylation indicates the presence of several activin-inducible sites of phosphorylation. It has been suggested that phosphorylation of residues in the juxtamembrane domain of T $\beta$ RI affects TGF $\beta$ signaling $[11,12]$. Mutation of the cognate residues in ALK4 (S168 and S174) to alanine had no effect on ALK4 activity (not shown). Identification of the other sites of ALK4 phosphorylation and determination of their functional significance are under way.

Acknowledgements: We thank Cole Zimmerman and Taju Karriaper for stimulating discussions and for comments on the manuscript. This research was supported by NIH Grants R01-GM50416 (to L.S.M.) and T32-HD07048 (to S.A.W.), and by funds from the Searle Scholars Program/The Chicago Community Trust (to L.S.M.).

\section{References}

[1] Mathews, L.S. (1994) Endocr. Rev. 15, 310-325.

[2] Massagué, J. (1996) Cell 85, 947-950.

[3] Willis, S.A., Zimmerman, C.M., Li, L. and Mathews, L.S. (1996) Mol. Endocrinol. 10, 367-379.

[4] Attisano, L., Wrana, J.L., Montalvo, E. and Massagué, J. (1996) Mol. Cell. Biol. 16, 1066-1073.

[5] Wrana, J.L., Attisano, L., Wieser, R., Ventura, F. and Massagué, J. (1994) Nature 370, 341-347.

[6] Attisano, L., Cárcamo, J., Ventura, F., Weis, F.M.B., Massagué, J. and Wrana, J.L. (1993) Cell 75, 671-680.

[7] Sambrook, J., Fritsch, E.F. and Maniatis, T. (1989) Molecular Cloning: A Laboratory Manual, Cold Spring Harbor Laboratory Press, Cold Spring Harbor, NY. 
[8] Wilson, I.A., Niman, H.L., Houghten, R.A., Cherenson, A.R., Connolly, M.L. and Lerner, R.A. (1984) Cell 37, 767-778.

[9] Mathews, L.S. and Vale, W.W. (1993) J. Biol. Chem. 263, 1901319018.

[10] Boyle, W.J., van der Geer, P. and Hunter, T. (1991) Methods Enzymol. 201, 110-149.
[11] Saitoh, M., Nishitoh, H., Amagasa, T., Miyazono, K., Takagi, M. and Ichijo, H. (1996) J. Biol. Chem. 271, 2769-2775.

[12] Souchelnytskyi, S., Ten Dijke, P., Miyazono, K. and Heldin, C.H. (1996) EMBO J. 15, 6231-6240. 